\title{
CARACTERIZAÇÃO FÍSICO-QUÍMICA, BIOCONVERSÃO DE RESÍDUOS AGROINDUSTRIAIS E COMPARAÇÃO ENTRE MÉTODOS DE INOCULAÇÃO
}

\section{PHYSICAL-CHEMICAL CHARACTERIZATION, BIOCONVERSION OF AGROINDUSTRIAL WASTES AND COMPARISON OF METHODS OF INOCULATION}

\begin{abstract}
Alessandra Zarpellon Teixeira ${ }^{1}$; Mariana Machado Fidelis do Nascimento ${ }^{2}$; Sabrina Ávila Rodrigues ${ }^{3}$; José Luiz Ferreira da Trindade ${ }^{4}$

1,2,3,4 Universidade Tecnológica Federal do Paraná - UTFPR - Ponta Grossa - Brasil ale_zt@yahoo.com.br

${ }^{3}$ Universidade Federal do Paraná - UFPR - Curitiba - Brasil marifideliss@ hotmail.com
\end{abstract}

\section{Resumo}

Uma alternativa de agregar valor a resíduos agroindustriais seria a bioconversão utilizando microrganismos, principalmente os fungos. O objetivo deste trabalho foi realizar um estudo acerca de alguns resíduos agroindustriais lignocelulósicos, analisando suas principais características físico-químicas e determinando qual apresenta maior potencial para ser utilizado como substrato em processos de biodegradação fúngica. A fibra do coco verde mostrou-se com grande potencial para ser utilizado como substrato em processos de bioconversão. Constatou que quanto menor o tamanho das fibras nos recipientes de fermentação, maior e mais eficaz é o processo de degradação. Realizou-se também uma comparação entre métodos de inoculação e adição de soluções de sacarose de distintas concentrações, constando que a inoculação líquida e a solução de sacarose a $10 \%$ foram bastante favorável e com melhores resultados para a degradação. $O$ "mix" de fibra de coco e resíduo da indústria cervejeira mostrou-se como excelente substrato com $81 \%$ de degradação em 42 dias.

Palavras-chave: resíduos agroindustriais, bioconversão, degradação

\section{Introdução}

Setores como o de alimentos e o agroindustrial tem uma grande geração de resíduos, muitas vezes com problemas de disposição final e com alto potencial poluente (VÉRAS, 2002). Nos últimos anos, há um interesse crescente no uso eficiente de diversos resíduos agroindustriais. Vários bioprocessos têm sido desenvolvidos utilizando estes materiais como substrato para a produção de diversas moléculas com alto valor agregado, tais como proteínas microbianas, ácidos orgânicos, etanol, enzimas e metabólitos secundários biologicamente ativos (ALEXANDRINO et al., 2007). 
A maioria desses resíduos agroindustriais são compostos de celulose, hemicelulose e lignina e são conhecidos como resíduos agroindustriais lignocelulósicos (REGINATTO, 1992). Uma alternativa de agregar valor a esses resíduos seria a bioconversão utilizando microrganismos, principalmente os fungos (VILLAS BOAS e ESPÓSITO, 2000). A bioconversão de resíduos agroindustriais se dá através de diversas técnicas, tal como, a fermentação em estado sólido (FES), de fácil operacionalização, pois não exige que os resíduos sejam previamente tratados o que, do ponto de vista industrial, torna-se um aspecto interessante (SILVA, 2006). Vários grupos de microrganismos podem crescer em substratos sólidos, porém os fungos filamentosos são os utilizados na maioria dos casos, devido às suas propriedades fisiológicas, enzimológicas e bioquímicas (SOCCOL et al., 1994).

O cogumelo Lentinula edodes, conhecido como shiitake, é classificado como pertencente à classe de Basidiomycetes, ordem Agaricales, família Agaricaceae (REGINA e BROETTO, 2005). Por ser apto a utilizar lignina, celulose e hemicelulose como fonte de carbono e nutrientes, o shiitake pode ser cultivado em uma grande variedades de resíduos agrícolas (ROSSI, 1999).

Buscando conhecer características físico-químicas específicas de alguns resíduos agroindustriais, realizou-se primeiramente, neste estudo, a análise de quatro diferentes matériasprima. Baseadas nos resultados encontrados nestas análises foram realizadas as fermentações iniciais e então uma otimização da bioconversão da fibra de coco verde (Cocos nucifera) por Lentinula edodes, através da comparação entre métodos de inoculação e do uso de soluções de sacarose, com diferentes concentrações, no desenvolvimento micelial inicial do fungo no substrato.

\section{Material e Métodos}

O fungo utilizado para o estudo compreendeu a cepa Lentinula edodes, disponível no acervo de fungos do laboratório de Microbiologia. Foram analisadas amostras de quatro diferentes resíduos: Resíduo de uma indústria cervejeira da região dos Campos Gerais, composto basicamente de gritz e cevada; Talos de trigo da variedade BRS220 (T2) produzidos em uma fazenda da região de Maringá cultivado com 50\% de adubo, uréia mais o inoculante (Azospirillum brasilienses); Talos de trigo da variedade BRS220 produzidos em uma fazenda da região de Maringá (T5), cultivado somente com o inoculante e a Fibra do coco verde, obtidos em pontos de vendas de água de coco na região. Para o desenvolvimento da pesquisa utilizou-se os resíduos triturados e esterilizados, realizando triplicata para cada amostra. As análises físico-químicas para a caracterização dos resíduos agroindustriais estudados e a determinação de proteínas foram baseadas nas metodologias descritas no manual Instituto Adolfo Lutz, 2008 (IAL, 2008). A etapa seguinte consistiu na análise dos resultados obtidos para então se dar inicio as fermentações. O fungo utilizado foi mantido em 
Placas de Petri, contendo o meio de cultivo batata dextrose Agar esterilizado a $121^{\circ} \mathrm{C}$ por 15 min. Após o crescimento fúngico por 5 dias a $28^{\circ} \mathrm{C}$ em estufa, as placas foram refrigeradas a $4^{\circ} \mathrm{C}$. O micélio cultivado em Placas de Petri foi utilizado como inóculo aos substratos selecionados (EIRA, 1997).

A primeira fermentação avaliou o desenvolvimento do micélio em diferentes granulometria da fibra de coco e se procedeu em recipiente de vidro de $200 \mathrm{~mL}$ de volume e $8 \mathrm{~cm}$ de altura. A fibra de coco verde utilizada como substrato, foi distribuída nos vidros conforme o tamanho que foi cortada, variando de $12,5 \%$ a $100 \%$ do tamanho da altura do vidro. Foram realizadas triplicatas, de cada um dos diferentes tamanhos. Na segunda fermentação (experimento 2), a fibra de coco verde sofreu corte para que tivesse a dimensão de $1 \mathrm{~cm}$ aproximadamente (12,5\%), em seguida foi distribuída nos vidros, sendo 70 gramas do substrato em cada. Foram preparados 5 vidros para cada tipo de inoculação e adicionado de soluções de sacarose as concentrações variaram de 0 (sem adição de sacarose) a $20 \%$. Os frascos contendo a fibra foram autoclavados a $121^{\circ} \mathrm{C}$, por 15 minutos e resfriados a temperatura ambiente. Os frascos foram conduzidos para inoculação, de acordo com cada método, com proximidade à chama.

Para inoculação padrão criou-se no centro do pote de vidro, um canal de inoculação onde o micélio formado em meio de cultivo BDA, foi introduzido. Já para a inoculação líquida, diluiu-se o micélio em meio cultivo $\mathrm{BDA}$, em água reagente II esterilizada, pipetou-se $10 \mathrm{~mL}$ do inóculo formado e inoculou-se por todo o substrato de maneira uniforme. Os frascos foram incubados a temperatura de $28{ }^{\circ} \mathrm{C}$ durante 23 dias. Durante o período de fermentação foram realizadas umidificações, com o uso de seringas estéreis, água reagente II esterilizada e com proximidade da chama.

No experimento 3 foi elaborado um "mix" de fibra de coco verde e resíduo da indústria cervejeira, com a proporção de $80 \%$ de fibra de coco verde e $20 \%$ de resíduo cervejeiro. Os resíduos foram pesados em balança analítica, inseridos nos frascos de fermentação e adicionou-se $10 \mathrm{~mL}$ de solução de sacarose a $10 \%$, que posteriormente foram conduzidos para serem autoclavados a $121^{\circ} \mathrm{C}$ por $15 \mathrm{~min}$. Em seguida, os frascos foram resfriados e realizada inoculação líquida nas mesmas condições descritas acima. Foram realizadas umidificações durante o período de fermentação, com o uso de seringas estéreis, meio MPII esterilizado e água reagente II esterilizada com proximidade da chama.

\section{Resultados e Discussão}

Após a realização das análises dos quatro resíduos, uma tabela foi elaborada para representar os resultados das análises. A Tabela 1 indica um resumo dos resultados obtidos nas caracterizações: 
Tabela 1 - Caracterização físico- química dos resíduos

\begin{tabular}{llllllll}
\hline & Carbono orgânico & C/N & Nitrogênio & Umidade & Cinzas & Proteínas & Fibra bruta \\
\hline Gritz e cevada & $51,14 \%$ & $10,8 \%$ & $4,7 \%$ & $9,03 \%$ & $3,95 \%$ & $29,37 \%$ & $14,5 \%$ \\
Trigo T2 & $53,32 \%$ & $76,2 \%$ & $0,7 \%$ & $15,31 \%$ & $5,84 \%$ & $4,37 \%$ & $45,6 \%$ \\
Trigo T5 & $50,42 \%$ & $72 \%$ & $0,7 \%$ & $15,38 \%$ & $6,14 \%$ & $4,37 \%$ & $47,6 \%$ \\
Fibra de coco & $55,30 \%$ & $553 \%$ & $0,10 \%$ & $86,61 \%$ & $0,45 \%$ & $0,63 \%$ & $3,1 \%$ \\
Biomassa & & & & & & $4,4 \%$ & $33,3 \%$ \\
\hline
\end{tabular}

A determinação de umidade pode ser considerada como o ponto de partida para processos de biodegradação (ALBERTON, 2004). Um excesso de umidade pode impedir as trocas gasosas do micélio ou proporcionar a proliferação de antagonistas e parasitas, mas para que ocorra a frutificação a umidade deve estar entre $85 \%$ e 95\% (CHANG e MILES, 2004). Os valores de umidade obtidos tiveram diferença significativa, tendo a fibra do coco verde apresentado valor considerado ideal para a frutificação nos substratos $(86,61)$. O teor de proteína bruta pode ser diretamente relacionado com o potencial do substrato a ser utilizado. Nas análises realizadas, a amostra que apresentou maior teor de proteína bruta foi o resíduo da indústria cervejeira, com 29,37\% enquanto que, para a fibra de coco verde o teor de proteína bruta encontrada foi bem abaixo desse valor, apresentando 0,63\%. Pode-se considerar, portanto, que por possuir um baixo teor de proteína a fibra do coco verde tem grande potencial para degradação já que o teor de nitrogênio deve estar presente em quantidade mínima, pois altas concentrações de nitrogênio limitam a degradação da lignina presente no substrato. O resíduo da indústria cervejeira, apresentando alto valor protéico pode ser utilizado como suplementação para substratos compostos que necessitem de fonte de nitrogênio adicional para a sua colonização. Todo composto para cultivo de cogumelos tem, como regra geral a escolha de materiais volumosos e fibrosos, geralmente muito ricos em carbono e pobres em nitrogênio e fósforo (EIRA, 1997). Considerando que o substrato com maior potencial para o cultivo de cogumelos, deve apresentar alta quantidade de carbono, a fibra do coco verde apresentou-se com melhor resultado para esta análise. Com base nesta análise, pôde-se então determinar a relação carbono/nitrogênio, uma das principais análises para a escolha de um substrato. Um padrão de substrato enriquecido com relação $\mathrm{C} / \mathrm{N}$ entre 15 e 25/1, pode ser utilizado no sistema de cultivo de cogumelos sob condições axênicas (EIRA, 2004). Como em todas as amostras, os valores não se encontram neste padrão (Tabela 1), o ideal seria a incorporação de componentes concentrados (normalmente tortas e farelos), em quantidades adequadas para atingir a relação $\mathrm{C} / \mathrm{N}$ ideal. A textura do substrato interfere também na frutificação, portanto a importância da determinação de fibra bruta. Para as amostras analisadas a textura dos talos de trigo foi a que se mostrou bastante fibrosa, apresentando valores aproximados de 45\%, o que acaba otimizando o processo de biodegradação. 
Com base nos resultados das análises físico-químicas realizadas, deu-se sequência ao trabalho com a realização da fermentação no estado sólido. O resíduo selecionado foi a fibra de coco verde já que a mesma, quando comparada com os outros resíduos analisados, apresentou características que apontaram para a possibilidade de sua utilização como substrato. Não foi estabelecido um número de dias fixos para a fermentação, o fim da fermentação deu-se quando se percebeu que não havia maiores progressões no desenvolvimento do micélio, e o mesmo havia tomado todo o substrato. Observou-se também, as condições de aeração dos substratos. Todos os experimentos apresentaram desenvolvimento micelial e distribuição dos corpos de frutificação significativa. No entanto, pôde-se observar ao final da fermentação, maior estágio de degradação no experimento de fibra de coco $12,5 \%$, podendo ser considerada como principal influência o menor tamanho, portanto maior superfície de contato do substrato com o oxigênio presente no interior do recipiente.

A produção micelial, no substrato e a produção de proteína é variável de acordo com o microrganismo, com o substrato e com as condições de culturas aplicadas (LIMA et al., 1975). Neste trabalho o cultivo de Lentinula edodes, no experimento 12,5\% proporcionou um enriquecimento de mais de $100 \%$ no teor de proteína e no teor de fibra bruta. Os valores de fibra bruta expressam praticamente somente a quantidade de celulose presente nos corpos de frutificação, sendo que os mesmos são constituídos predominantemente por hemicelulose e celulose (SCARIOT e MARLEI, 2000), portanto, o aumento no teor de fibra bruta pode ter relação com o surgimento de corpos de frutificação no substrato, já que desta maneira, os valores para celulose também seriam afetados.

No experimento 2 constatou-se que a inoculação líquida se mostrou um método eficiente, pois reduziu o tempo de colonização do substrato pelo microrganismo. Chang e Miles (1993) citado por Silveira et al. (2008), afirmam que a substituição do inóculo sólido por líquido pode reduzir os custos de laboratório e tornar mais fácil a técnica de inoculação, bem como pode ser usado para a proliferação do micélio em suporte sólido ou inoculado diretamente no substrato de produção. O uso da sacarose como coadjuvante no início da fermentação foi favorável e as concentrações com resultados mais satisfatórios para o desenvolvimento do micélio foram sem adição de sacarose ou adicionados de 5 e $10 \%$ do mesmo, tanto na inoculação líquida quanto na inoculação padrão. Nas outras concentrações, o aspecto visual do fungo era ressecado, característica que pode ser atribuída à alta concentração de sacarose no meio. Segundo Karunanandaa et al. (1995) no decorrer do processo de colonização de substratos lignocelulósicos, os fungos preferencialmente convertem os polissacarídeos mais facilmente digeridos em açúcares de baixo peso molecular, utilizando-os para seu metabolismo primário. Outro estudo realizado por Leatham (1986), citado por Rossi et al. 
(2001) demonstrou haver necessidade de fornecimento de açúcar para aumentar a atividade lignocelulolítica.

O "mix" formulado no experimento 3 mostrou-se interessante para ser utilizado em processos de bioconversão, principalmente, devido à suplementação do substrato com resíduo cervejeiro, pois favoreceu a miceliação, manteve o vigor do fungo Lentínula edodes e acelerou o processo de bioconversão. O estudo realizado por Sing e Verna (1996), citado por Pedra e Marino (2006), demonstrou que houve aumento significativo de vigor com a suplementação à base de farelos. A suplementação de substratos lignocelulósicos com farelos altera a relação C:N do substrato favorecendo o crescimento micelial mais vigoroso. Fasini e Kadiri (1993), citado por Marino e Abreu (2009) atribuíram aos suplementos à base de farelos um efeito estimulante para o crescimento micelial, relacionado à presença de carboidratos, aminoácidos e minerais.

O comparativo do percentual de biomassa degrada e a solução umidificante utilizada pode ser observado na Tabela 2.

Tabela 2- Percentual de biomassa degradada em relação à solução umidificante

\begin{tabular}{cc}
\hline & \% de biomassa degradada \\
\hline Água esterilizada & $76 \%$ \\
Meio MPII & $81 \%$ \\
\hline
\end{tabular}

O percentual de biomassa degradada foi bastante elevado nos dois experimentos, mas o uso de diferentes soluções umidificantes demonstrou que o experimento umidificado pelo meio de cultura MPII, apresentou percentual de biomassa degradada levemente maior do que o experimento, apenas umidificado com água esterilizada. Esse percentual maior de degradação no experimento com Meio MPII como solução umidificante, pode ser atribuído aos compostos formadores do meio, em função do meio ser rico em proteínas e minerais, compostos que podem ter favorecido o desenvolvimento do microrganismo e a degradação do substrato.

\section{Conclusão}

Considerando os resultados do presente trabalho, concluiu-se que processos de biodegradação fúngica podem ser considerados uma importante alternativa para se agregar valor a resíduos agroindustriais lignocelulósicos. Todos os resíduos agroidustriais analisados apresentaram características que lhes conferiram potencial para serem utilizados como substratos em fermentações ou como suplementação de meios utilizados para fermentações.

No entanto, a fibra de coco verde apresentou propriedades físico-químicas específicas que possibilitaram, juntamente com variáveis como temperatura e tempo de fermentação, a obtenção de resultados satisfatórios no processo de fermentação. A escolha do tratamento dos substratos, 
diferenciados entre si, pelos seus diferentes tamanhos, possibilitou melhor visualização do processo. Pode-se estabelecer uma comparação quanto à aeração no interior do recipiente. Apresentou-se como melhor meio adaptado para degradação, o experimento elaborado com a fibra de coco verde em tamanho $12,5 \%$, apresentando um alto grau de biodegradação com aumento significativo no percentual de proteínas e fibra bruta pós-fermentação.

A inoculação líquida é um método eficiente e a uniformidade apresentada na distribuição do micélio no substrato foi devida ao seu uso. O uso de soluções de sacarose mostrou-se favorável, com melhor resultado nos experimentos sem adição de sacarose ou adicionados 5 e $10 \%$ do mesmo. Os demais frascos, com solução de sacarose a 15 e $20 \%$ apresentaram aspecto visual ressecado devido à alta concentração desse açúcar. $\mathrm{O}$ "mix" de fibra de coco e resíduo da indústria cervejeira demonstrou-se um excelente substrato, visto que ocorreu $81 \%$ de degradação do substrato em apenas 42 dias. O meio MPII mostrou-se uma boa solução umidificante devido a sua constituição muito rica.

\begin{abstract}
An alternative to add value to agro-industrial wastes would be the bioconversion using microorganisms, mainly fungi. The objective of this work was realize a study about of some lignocellulosic agro-industrial wastes, analyzing their main physico-chemical characteristics and determining which has greater potential to be used as the substrate in fungal biodegradation processes. The green coconut fiber was shown with great potential to be used as the substrate for bioconversion processes. Found that as smaller the size of the fibers on fermentation vessels, larger and more efficient is the process of degradation. Realized also a comparison between methods of inoculation and addition of sucrose solutions of distinct concentrations, being the liquid inoculation and sucrose solution at $10 \%$ were very favorable and with better results for degradation. The mix of coconut fiber and waste from the brewing industry showed to be excellent substrate with $81 \%$ degradation in 42 days.
\end{abstract}

Key-words: agro-industrial wastes, bioconversion, degradation

\title{
Referências
}

ALBERTON, L. R. Produção de xilanas em resíduos agroindustriais por Streptomyces viridosporus T7A e aplicação do extrato bruto em veterinária. Curitiba: UFPR, 2004.

ALEXANDRINO, A.M.; FARIA, G.; SOUZA, C.G.M.; PERALTA, R.M. Aproveitamento do resíduo de laranja para a produção de enzimas lignocelulolíticas por Pleurotus ostreatus. Ciência e Tecnologia de Alimentos, v. 27, n. 02, p. 364-368, 2007. DOI: 10.1590/S0101-20612007000200026

CHANG, S. T.; MILES, P. G. Mushrooms: Cultivation, Nutricional Value Medicinal Effect and Envirommental Impact. 2 ed. CRC Press: Boca Raton, 2004.

EIRA, A. F.; Minhoni, M. T. A. Manual teórico-prático do cultivo de cogumelos comestíveis. Botucatu: Fundação de Pesquisa Agropecuária e Florestais, 1997. 75p.

EIRA, A.F. Fungos comestíveis. In: Esposito; Azevedo, J. L. Fungos: uma introdução à biologia, bioquímica e biotecnologia, Caxias do Sul: EDUCS, 2004. Cap. 12. 
INSTITUTO ADOLFO LUTZ. Métodos físico-químicos para análise de alimentos. Coordenadores: ZENEBON, O.; PASCUET, N. S; TIGLEA, P. 1 ed digital. São Paulo:Instituto Adolfo Lutz, 2008. 1020 p.

KARUNANANDAA, K.; VARGA, G.A; AKIN, D.E; RIGSBY, L.L.; ROYSE, D.J. Botanical fractions of rice straw colonized by white rot fungi changes in chemical composition and structure. Animal feed science and technology, Amsterdam, v.55, n.3-4, out, p. 179-199, 1995.

LIMA, U.A., AQUARONE, E., BORZANI, W. Biotecnologia: Tecnologia das fermentações. São Paulo, Edgard Blucher, vol. 1, 1975.

MARINO, R. H.; ABREU, L. D. de. Cultivo do cogumelo Shiitake em resíduo de coco suplementado com farelo de trigo e/ou arroz. Revista Brasileira de Ciências Agrárias, Recife, v.4, n.1, p.11-16, jan. - mar., 2009.

PEDRA, W.N.; MARINO, R.H. Cultivo axênico de Pleurotus spp. em serragem da casca de coco (Cocos nucifera linn.) suplementada com farelo de arroz e/ou de trigo. Arquivos do Instituto Biológico, São Paulo, v.73, n.2, p.219-225, abr./jun., 2006.

REGINA, M; BROETTO, F. Atividades de enzimas oxidativas do Lentinula edodes em meio de cultura liquida de subprodutos energéticos. Energia Agrícola. v. 20, n. 1, Botucatu, 2005.

REGINATTO, V. Estudo das enzimas produzidas por Trichoderma longibrachiatum responsáveis pela degradação de materiais celulósicos. Campinas, 1992. 147p. Dissertação de mestrado - Faculdade de Engenharia de Alimentos, Universidade Estadual de Campinas.

ROSSI, I. H.; MONTEIRO, A. C., MACHADO, J. O. Desenvolvimento micelial de Lentinula edodes como efeito da profundidade e suplementação do substrato. Pesquisa agropecuária brasileira, Brasília, v. 36, n. 6, p. 887-891, jun. 2001.

ROSSI, I.H. Suplementação de bagaço de cana para cultivo axênico do cogumelo shiitake (Lentinula edodes). UNESP - Dissertação de Mestrato. Jaboticabal, 1999.

SCARIOT, MARLEI R.. Composição química de cogumelos comestíveis cultivados em resíduo de algodão (Gossypium hirsutum L. ). Acta Scientiarum, v. 22, n. 2, p. 317-320, 2000.

SILVA, G. Aproveitamento Biotecnológico de Resíduos Agroindustriais na Produção de Glucoamilase. Blumenau, 2006. Dissertação de mestrado - Universidade Regional de Blumenau - FURB.

SILVEIRA, M. L. L. FURLAN, S. A. NINOW, J. L. Development of an alternative technology for the oyster mushroom production using liquid inoculum. Ciência e Tecnologia em Alimentos, v. 28, n. 04, p. 858-862, 2008. DOI: 10.1590/S0101-20612008000400014

SOCCOL, C.R., MARIN, B., RAIMBAULT, M., LEBEAULT, J-M. Breeding and growth of Rhizopus in raw cassava by solid state fermentation. Applied Microbiology Biotechnology, v. 41, p. 330- 336, 1994. DOI: $10.1007 / \mathrm{BF} 00221228$

VÉRAS, A. A indústria de alimentos e o meio ambiente: Impacto ambiental da indústria de alimentos. ITAL, Campinas, 2002.

VILLAS BOAS, S.G.; ESPÓSITO, E. Bioconversão do bagaço de maçã. Biotecnologia, ciência e desenvolvimento. Brasília, v. 3, n. 14, p. 38-42, 2000.

Trabalho selecionado para apresentação oral durante a VIII SETAL- Semana de Tecnologia de Alimentos- Câmpus Ponta Grossa- Universidade Tecnológica Federal do Paraná- 01 a 03 de junho de 2011. Suplemento especial da RBTA. 